\title{
VOLUME INEQUALITIES FOR ISOTROPIC MEASURES
}

\author{
Erwin Lutwak, Deane Yang, and Gaoyong Zhang \\ Department of Mathematics \\ Polytechnic University \\ Brooklyn, NY 11201
}

\begin{abstract}
A direct approach to Ball's simplex inequality is presented. This approach, which does not use the Brascamp-Lieb inequality, also gives Barthe's characterization of the simplex for Ball's inequality and extends it from discrete to arbitrary measures. It also yields the dual inequality, along with equality conditions, and it does both for arbitrary measures.
\end{abstract}

A non-negative Borel measure $Z$ on the unit sphere $S^{n-1}$ is said to be isotropic if, when viewed as a mass distribution on $S^{n-1}$, it has the same moment of inertia about all lines through the origin. The convex hull of the support of $Z$ is a convex body $Z_{\infty}$ in $\mathbb{R}^{n}$. Ball used his elegant reformulation of the Brascamp-Lieb inequality to obtain the sharp upper bound for the volume of the polar $Z_{\infty}^{*}$ of the body $Z_{\infty}$. Ball showed how this could be combined with John's characterization of the ellipsoid of maximal volume contained in a convex body, to prove his celebrated reverse isoperimetric inequality [2]. Recently, Barthe [5] found a beautiful new approach to establishing the Brascamp-Lieb inequality. Barthe's approach has the added advantage that it yields the equality conditions for Ball's reformulation of the Brascamp-Lieb inequality. Armed with these equality conditions, Barthe was able to show that, when $Z$ is discrete, the simplex is the unique extremal for Ball's inequality.

In this paper we give a direct proof that for and only for the simplex is the sharp upper bound for the volume of $Z_{\infty}^{*}$ attained. The advantage of our approach is that it allows us to establish Ball's simplex inequality, along with Barthe's equality conditions, for measures which are not necessarily discrete. Another advantage of our approach is that it allows us to establish the analogous results for the body $Z_{\infty}$

The equality conditions of the Brascamp-Lieb inequality are complicated. Our approach is based on a continuous version of the Ball-Barthe inequality that has simple and easily stated equality conditions.

We have attempted to write an article that is self-contained. We have given very detailed proofs (but did not reprove the Ball-Barthe inequality and refer the reader to [28]). Although the questions we address (here and in [28]) arise naturally within the Brunn-Minkowski-Firey theory (see, e.g., [7$10,13-27,29-32,34,35,37])$ none of the machinery of the theory is used.

The ideas and techniques of Ball and Barthe play a critical role in this paper. It would be impossible to overstate our reliance on their work.

If $Z$ is a finite nonnegative Borel measure on the unit sphere $S^{n-1}$ and is not concentrated on a closed hemisphere of $S^{n-1}$, then define the convex body $Z_{\infty}$ in $\mathbb{R}^{n}$ as the convex hull of the support of $Z$; i.e.,

$$
Z_{\infty}=\operatorname{conv}(\operatorname{supp} Z) .
$$

1991 Mathematics Subject Classification. MR 52A40.

Research supported, in part, by NSF Grant DMS-0104363 and DMS-0405707 
Note that the convex body $Z_{\infty}$ contains the origin in its interior (since $\operatorname{supp} Z$ is not contained in a closed hemisphere of $S^{n-1}$ ). The polar body $Z_{\infty}^{*}$ of $Z_{\infty}$ is given by

$$
Z_{\infty}^{*}=\left\{x \in \mathbb{R}^{n}: x \cdot v \leq 1 \text { for all } v \in \operatorname{supp} Z\right\},
$$

where $x \cdot v$ denotes the standard inner product of $x$ and $v$ in $\mathbb{R}^{n}$. Note that since $Z_{\infty}$ is by definition contained in the unit ball, $B$, it follows that $B \subseteq Z_{\infty}^{*}$.

In addition to its denoting absolute value, we shall use $|\cdot|$ to denote the standard Euclidean norm on $\mathbb{R}^{n}$, on occasion the absolute value of the determinant of an $n \times n$ matrix, and often to denote $n$-dimensional volume.

A finite nonnegative Borel measure $Z$ on $S^{n-1}$ is said to be isotropic if

$$
|x|^{2}=\int_{S^{n-1}}|x \cdot u|^{2} d Z(u),
$$

for all $x \in \mathbb{R}^{n}$. Note that it is impossible for an isotropic measure to be concentrated on a proper subspace of $\mathbb{R}^{n}$. The centroid of the measure $Z$ is defined as

$$
\frac{1}{Z\left(S^{n-1}\right)} \int_{S^{n-1}} u d Z(u)
$$

The purpose of this paper is to establish the following two theorems.

Theorem 1. If $Z$ is an isotropic measure on $S^{n-1}$ whose centroid is at the origin, then

$$
\left|Z_{\infty}^{*}\right| \leq n^{n / 2}(n+1)^{(n+1) / 2} / n !
$$

with equality if and only if $Z_{\infty}$ is a regular simplex inscribed in $S^{n-1}$.

The volume inequality was proved by Ball [2]. For discrete measures, the equality conditions were obtained by Barthe [5].

The following dual to the inequality in Theorem 1 was anticipated by Ball [2] and established for discrete measures by Barthe [4].

Theorem 2. If $Z$ is an isotropic measure on $S^{n-1}$ whose centroid is at the origin, then

$$
\left|Z_{\infty}\right| \geq(n+1)^{(n+1) / 2} n^{-n / 2} / n !,
$$

with equality if and only if $Z_{\infty}$ is a regular simplex inscribed in $S^{n-1}$.

Write $\left\{e_{1}, \ldots, e_{n}\right\}$ for the standard orthonormal basis of $\mathbb{R}^{n}$. If, in the definition (3) of an isotropic measure, we set $x=e_{j}$ and sum over all $j$ we immediately see that the total mass of an isotropic measure on $S^{n-1}$ is always $n$; i.e.,

$$
Z\left(S^{n-1}\right)=n
$$

We shall also make use of the simple observation that if $\operatorname{supp} Z=\left\{u_{1}, \ldots, u_{n}\right\} \subset S^{n-1}$, then the vectors $u_{1}, \ldots, u_{n}$ must be orthogonal. To see this first note that since $Z$ is isotropic, from (3) we have for all $x \in \mathbb{R}^{n}$,

$$
\sum_{i=1}^{n} c_{i}\left|x \cdot u_{i}\right|^{2}=|x|^{2},
$$

where $c_{i}=Z\left(\left\{u_{i}\right\}\right)>0$. Taking $x=u_{j}$, gives

$$
\sum_{i=1}^{n} c_{i}\left|u_{j} \cdot u_{i}\right|^{2}=1
$$

This shows that $c_{j} \leq 1$. But from (4) we know $\sum_{i=1}^{n} c_{i}=n$ and hence, $c_{j}=1$. Thus, $\left|u_{j} \cdot u_{i}\right|=0$ for $j \neq i$. 
If $\nu$ is a Borel measure on $S^{n-1}$, let $|f: \nu|_{p}$ denote the standard $L_{p}$ norm of the function $f$, with respect to $\nu$; i.e., for $1 \leq p<\infty$

$$
|f: \nu|_{p}=\left(\int_{S^{n-1}}|f(u)|^{p} d \nu(u)\right)^{1 / p} .
$$

The support function $h_{K}: S^{n-1} \rightarrow \mathbb{R}$ of a convex body $K$ in $\mathbb{R}^{n}$ is defined by

$$
h_{K}(u)=\max \{u \cdot x: x \in K\}
$$

for $u \in S^{n-1}$. For each measure $Z$ on $S^{n-1}$ the convex body $Z_{\infty} \subseteq B$ may be defined as the convex body whose support function is given by

$$
h_{Z_{\infty}}(u)=\max \{u \cdot v: v \in \operatorname{supp} Z\},
$$

for $u \in S^{n-1}$. From (2) we see that

$$
\operatorname{int} Z_{\infty}^{*}=\left\{x \in \mathbb{R}^{n}: x \cdot v<1 \text { for all } v \in \operatorname{supp} Z\right\} .
$$

The following continuous version of the Ball-Barthe inequality was proved in [28] as a direct consequence of the Hölder inequality.

Ball-Barthe lemma. If $\nu$ is an isotropic measure on $S^{n-1}$ and $t: S^{n-1} \rightarrow(0, \infty)$ is continuous, then

$$
\operatorname{det} \int_{S^{n-1}} t(v) v \otimes v d \nu(v) \geq \exp \left\{\int_{S^{n-1}} \log t(v) d \nu(v)\right\}
$$

with equality if and only if $t\left(v_{1}\right) \cdots t\left(v_{n}\right)$ is constant for linearly independent $v_{1}, \ldots, v_{n} \in \operatorname{supp}(\nu)$.

Here, $v \otimes v: \mathbb{R}^{n} \rightarrow \mathbb{R}^{n}$ is the rank 1 linear operator $x \mapsto(x \cdot v) v$.

For $t \in L_{1}(Z)$, define $t^{\circ} \in \mathbb{R}^{n}$ by

$$
t^{\circ}=\int_{S^{n-1}} u t(u) d Z(u) .
$$

We shall make use of the following trivial fact.

Lemma 1. If $Z$ is an isotropic measure on $S^{n-1}$ and $t \in L_{2}(Z)$, then

$$
\left|t^{\circ}\right| \leq|t: Z|_{2},
$$

with equality if and only if $t(u)=u \cdot t^{\circ}$ for almost all $u \in S^{n-1}$ with respect to the measure $Z$.

Proof. From the definition of $t^{\circ}$, the Cauchy-Schwarz inequality, and finally using the fact that $Z$ is isotropic we have

$$
\begin{aligned}
\left|t^{\circ}\right|^{2} & =t^{\circ} \cdot t^{\circ} \\
& =\int_{S^{n-1}}\left(t^{\circ} \cdot u\right) t(u) d Z(u) \\
& \leq \int_{S^{n-1}}\left|t^{\circ} \cdot u\right||t(u)| d Z(u) \\
& \leq|t: Z|_{2}\left[\int_{S^{n-1}}\left|t^{\circ} \cdot u\right|^{2} d Z(u)\right]^{\frac{1}{2}} \\
& =|t: Z|_{2}\left|t^{\circ}\right| .
\end{aligned}
$$

We shall require the following simple fact: 
Lemma 2. Suppose $Z$ is a measure on $S^{n-1}$ whose support is not contained in a closed hemisphere of $S^{n-1}$. If $t: S^{n-1} \rightarrow(0, \infty)$ is a continuous function such that $|t: Z|_{1}=1$, then $t^{\circ} \in \operatorname{int} Z_{\infty}$.

Proof. Suppose $v \in S^{n-1}$. Since $t>0$, and $\operatorname{supp} Z$ is not contained in a closed hemisphere of $S^{n-1}$, we have

$$
v \cdot t^{\circ}=\int_{S^{n-1}}(v \cdot u) t(u) d Z(u)<|t: Z|_{1} \max _{u \in \operatorname{supp} Z} v \cdot u=h_{Z_{\infty}}(v),
$$

for all $v \in S^{n-1}$, which gives the desired result that $t^{\circ} \in \operatorname{int} Z_{\infty}$.

We shall make use of Lemma 2 only in the following form: If $t: S^{n-1} \rightarrow(0, \infty)$ is continuous, then

$$
\int_{S^{n-1}} u t(u) d Z(u) / \int_{S^{n-1}} t(u) d Z(u) \in \operatorname{int} Z_{\infty} .
$$

We shall need the function $s: S^{n-1} \rightarrow S^{n} \subset \mathbb{R}^{n+1}=\mathbb{R}^{n} \times \mathbb{R}$, defined by

$$
s(u)=\left(-\frac{\sqrt{n}}{\sqrt{n+1}} u, \frac{1}{\sqrt{n+1}}\right),
$$

for each $u \in S^{n-1}$.

Following Ball's idea, we show how each isotropic measure $Z$ on $S^{n-1}$, whose centroid is at the origin, induces an isotropic measure $\bar{Z}$ on $S^{n}$. Define the measure $\bar{Z}$ on $S^{n}$, concentrated on the subsphere

$$
D=\left\{x \in S^{n}: x \cdot e_{n+1}=1 / \sqrt{n+1}\right\},
$$

by

$$
\int_{S^{n}} f d \bar{Z}=\int_{D} f d \bar{Z}=\frac{n+1}{n} \int_{S^{n-1}} f \circ s d Z
$$

for each continuous $f: S^{n} \rightarrow \mathbb{R}$.

To see that $\bar{Z}$ is an isotropic measure on $S^{n}$, i.e.,

$$
\int_{S^{n}}|y \cdot v|^{2} d \bar{Z}(v)=|y|^{2}
$$

for all $y \in \mathbb{R}^{n+1}$, note that from definition (8), the fact that $Z$ is isotropic, the fact that the centroid of $Z$ is at the origin, and (4), we have, for $y=(x, r) \in \mathbb{R}^{n+1}=\mathbb{R}^{n} \times \mathbb{R}$,

$$
\begin{aligned}
\int_{S^{n}}|y \cdot w|^{2} d \bar{Z}(w) & =\frac{n+1}{n} \int_{S^{n-1}}\left|(x, r) \cdot\left(-\frac{\sqrt{n}}{\sqrt{n+1}} u, \frac{1}{\sqrt{n+1}}\right)\right|^{2} d Z(u) \\
& =\int_{S^{n-1}}|x \cdot u|^{2} d Z(u)-\frac{2 r}{\sqrt{n}} x \cdot \int_{S^{n-1}} u d Z(u)+\frac{r^{2}}{n} \int_{S^{n-1}} d Z(u) \\
& =|x|^{2}+r^{2} \\
& =|y|^{2} .
\end{aligned}
$$

Since $\bar{Z}$ is an isotropic measure on $S^{n}$, from (4) we have

$$
\bar{Z}\left(S^{n}\right)=n+1 .
$$

Observe that since $\bar{Z}$ is isotropic, supp $\bar{Z}$ cannot be contained in a subspace of $\mathbb{R}^{n+1}$.

From the fact that $Z$ has its centroid at the origin and the fact that the plane of support of $\bar{Z}$ is orthogonal to $e_{n+1}$, and passes through $(0, \ldots, 0,1 / \sqrt{n+1})$, it is easily seen that the centroid of $\bar{Z}$ is $e_{n+1} / \sqrt{n+1}$; i.e.,

$$
\int_{S^{n}} w d \bar{Z}(w)=\sqrt{n+1} e_{n+1}
$$


Indeed, from definition (8), the fact that the centroid of $Z$ is at the origin, and (4), we have for each $y=(x, r) \in \mathbb{R}^{n+1}=\mathbb{R}^{n} \times \mathbb{R}$,

$$
\begin{aligned}
y \cdot \int_{S^{n}} w d \bar{Z}(w) & =\frac{n+1}{n} \int_{S^{n-1}}(x, r) \cdot\left(-\frac{\sqrt{n}}{\sqrt{n+1}} u, \frac{1}{\sqrt{n+1}}\right) d Z(u) \\
& =-\frac{\sqrt{n+1}}{\sqrt{n}} x \cdot \int_{S^{n-1}} u d Z(u)+\frac{\sqrt{n+1}}{n} r \int_{S^{n-1}} d Z(u) \\
& =\sqrt{n+1} r \\
& =y \cdot\left(\sqrt{n+1} e_{n+1}\right) .
\end{aligned}
$$

We now prove:

Theorem 1. If $Z$ is an isotropic measure on $S^{n-1}$ whose centroid is at the origin, then

$$
\left|Z_{\infty}^{*}\right| \leq n^{n / 2}(n+1)^{(n+1) / 2} / n !
$$

with equality if and only if $Z_{\infty}$ is a regular simplex inscribed in $S^{n-1}$.

Proof. Define the strictly increasing function $\phi:(0, \infty) \rightarrow \mathbb{R}$ by

$$
\int_{0}^{t} e^{-s} d s=\frac{1}{\sqrt{\pi}} \int_{-\infty}^{\phi(t)} e^{-s^{2}} d s
$$

Note that $\phi^{\prime}>0$ and that for all $t>0$, we have

$$
-t=-\log \sqrt{\pi}-\phi(t)^{2}+\log \phi^{\prime}(t) .
$$

Define the open cone $C \subset \mathbb{R}^{n+1}=\mathbb{R}^{n} \times \mathbb{R}$ by

$$
C=\bigcup_{r>0}\left(\frac{r}{\sqrt{n}} \operatorname{int} Z_{\infty}^{*}\right) \times\{r\} .
$$

Now $y=(x, r) \in C$ if and only if $\frac{\sqrt{n}}{r} x \in \operatorname{int}\left(Z_{\infty}^{*}\right)$, and from (5) we see that this is equivalent to $x \cdot u<\frac{r}{\sqrt{n}}$ for all $u \in \operatorname{supp} Z$. Using (7) we may rewrite this as

$$
y \in C \quad \text { if and only if } \quad y \cdot s(u)>0, \text { for all } u \in \operatorname{supp} Z \text {. }
$$

Following Barthe's idea, define a transformation $T: C \rightarrow \mathbb{R}^{n+1}$ by

$$
T y=\int_{S^{n}} w \phi(y \cdot w) d \bar{Z}(w)
$$

or, by (8), equivalently,

$$
T y=\frac{n+1}{n} \int_{S^{n-1}} s(u) \phi(y \cdot s(u)) d Z(u),
$$

for each $y \in C$. From (14) we see that $y \cdot s(u)$ is in the domain of $\phi$ for each $y \in C$ and each $u \in \operatorname{supp} Z$.

From (15) it follows that the differential of $T$ is given by

$$
d T(y)=\int_{S^{n}} \phi^{\prime}(y \cdot w) w \otimes w d \bar{Z}(w)
$$

for each $y \in C$. Thus, for each $z \in \mathbb{R}^{n+1}$,

$$
z \cdot d T(y) z=\int_{S^{n}} \phi^{\prime}(y \cdot w)|w \cdot z|^{2} d \bar{Z}(w) .
$$


Since $\phi^{\prime}>0$ and $\bar{Z}$ is not concentrated on a proper subspace of $\mathbb{R}^{n+1}$, we conclude that the matrix $d T(y)$ is positive definite for each $y \in C$. Hence, a simple application of the mean value theorem shows that $T: C \rightarrow \mathbb{R}^{n+1}$ is globally $1-1$.

From (12), (10), (17) and the Ball-Barthe inequality, (15) and Lemma 1, and making the change of variables $z=T y$, we have

$$
\begin{aligned}
\int_{C} & \exp \left\{-\int_{S^{n}} y \cdot w d \bar{Z}(w)\right\} d y \\
& =\int_{C} \exp \left\{-\int_{S^{n}}\left(\phi(y \cdot w)^{2}-\log \phi^{\prime}(y \cdot w)+\log \sqrt{\pi}\right) d \bar{Z}(w)\right\} d y \\
& =\pi^{-\frac{n+1}{2}} \int_{C} \exp \left\{-\int_{S^{n}} \phi(y \cdot w)^{2} d \bar{Z}(w)\right\} \exp \left\{\int_{S^{n}} \log \phi^{\prime}(y \cdot w) d \bar{Z}(w)\right\} d y \\
& \leq \pi^{-\frac{n+1}{2}} \int_{C} \exp \left\{-\int_{S^{n}} \phi(y \cdot w)^{2} d \bar{Z}(w)\right\}|d T(y)| d y \\
& \leq \pi^{-\frac{n+1}{2}} \int_{C} \exp \left\{-|T y|^{2}\right\}|d T(y)| d y \\
& \leq \pi^{-\frac{n+1}{2}} \int_{\mathbb{R}^{n+1}} e^{-|z|^{2}} d z \\
& =1 .
\end{aligned}
$$

On the other hand, from (11) and (13), we have

$$
\begin{aligned}
\int_{C} \exp \left\{-\int_{S^{n}} y \cdot w d \bar{Z}(w)\right\} d y & =\int_{C} \exp \left\{-y \cdot \int_{S^{n}} w d \bar{Z}(w)\right\} d y \\
& =\int_{C} \exp \left\{-\sqrt{n+1} y \cdot e_{n+1}\right\} d y \\
& =\int_{0}^{\infty} \int_{\frac{r}{\sqrt{n}} \operatorname{int}\left(Z_{\infty}^{*}\right)} e^{-\sqrt{n+1} r} d x d r \\
& =\left|Z_{\infty}^{*}\right| \int_{0}^{\infty}\left(\frac{r}{\sqrt{n}}\right)^{n} e^{-\sqrt{n+1} r} d r \\
& =\left|Z_{\infty}^{*}\right| n^{-n / 2} n !(n+1)^{-(n+1) / 2}
\end{aligned}
$$

which gives the desired inequality.

Suppose there is equality in the inequality of our theorem. Since $\bar{Z}$ is not concentrated on a proper subspace of $\mathbb{R}^{n+1}$, there are linearly independent unit vectors $w_{1}, \ldots, w_{n+1} \in \operatorname{supp} \bar{Z}$. Assume there is a different unit vector $w_{0} \in \operatorname{supp} \bar{Z}$. Write $w_{0}=\lambda_{1} w_{1}+\cdots+\lambda_{n+1} w_{n+1}$. At least one coefficient, say $\lambda_{1}$, is not zero. Since $w_{0}, w_{2}, \ldots, w_{n+1}$ are linearly independent, the equality conditions of the Ball-Barthe inequality imply that

$$
\phi^{\prime}\left(y \cdot w_{1}\right) \cdots \phi^{\prime}\left(y \cdot w_{n+1}\right)=\phi^{\prime}\left(y \cdot w_{0}\right) \phi^{\prime}\left(y \cdot w_{2}\right) \cdots \phi^{\prime}\left(y \cdot w_{n+1}\right),
$$

for all $y \in C$. But $\phi^{\prime}>0$, and hence we have

$$
\phi^{\prime}\left(y \cdot w_{1}\right)=\phi^{\prime}\left(y \cdot w_{0}\right),
$$

for all $y \in C$. Differentiating both sides with respect to $y$ shows that

$$
\phi^{\prime \prime}\left(y \cdot w_{1}\right) w_{1}=\phi^{\prime \prime}\left(y \cdot w_{0}\right) w_{0}
$$

for all $y \in C$. Since there exists $y \in C$ such that $\phi^{\prime \prime}\left(y \cdot w_{1}\right) \neq 0$ it follows that $w_{0}= \pm w_{1}$. But $Z$ is supported inside an open hemisphere of $S^{n}$, so $w_{0}=w_{1}$. 
Hence equality in our inequality implies that $\operatorname{supp} \bar{Z}=\left\{w_{1}, \ldots, w_{n+1}\right\}$. Since $\bar{Z}$ is isotropic, $w_{1}, \ldots, w_{n+1}$ are orthogonal. But $w_{i} \perp w_{j}$ implies $u_{i} \cdot u_{j}=1 / n$, for $i \neq j$, and thus $\operatorname{supp} Z$ consists of the vertices of a regular simplex inscribed in $S^{n-1}$.

When $Z$ is a discrete measure, the inequality of Theorem 1 was proved by Ball [2]. For discrete measures, the equality conditions of Theorem 1 were obtained by Barthe [5].

The inequality of the following theorem was anticipated by Ball [2] and, for discrete measures, established by Barthe [4].

Theorem 2. If $Z$ is an isotropic measure on $S^{n-1}$ whose centroid is at the origin, then

$$
\left|Z_{\infty}\right| \geq(n+1)^{(n+1) / 2} n^{-n / 2} / n !,
$$

with equality if and only if $Z_{\infty}$ is a regular simplex inscribed in $S^{n-1}$.

Proof. Define the strictly increasing function $\phi: \mathbb{R} \rightarrow(0, \infty)$ by

$$
\int_{0}^{\phi(t)} e^{-s} d s=\frac{1}{\sqrt{\pi}} \int_{-\infty}^{t} e^{-s^{2}} d s .
$$

Note that $\phi^{\prime}>0$, and that for all $t \in \mathbb{R}$, we have

$$
t^{2}=\phi(t)-\log \phi^{\prime}(t)-\log \sqrt{\pi} .
$$

Define a transformation $T: \mathbb{R}^{n+1} \rightarrow \mathbb{R}^{n+1}$ by

$$
T y=\int_{S^{n}} w \phi(y \cdot w) d \bar{Z}(w),
$$

or, by (8), equivalently by

$$
T y=\frac{n+1}{n} \int_{S^{n-1}} s(u) \phi(y \cdot s(u)) d Z(u) .
$$

Define the open cone $C \subset \mathbb{R}^{n+1}=\mathbb{R}^{n} \times \mathbb{R}$ by

$$
C=\bigcup_{r>0}\left(-r \sqrt{n} \operatorname{int} Z_{\infty}\right) \times\{r\} .
$$

Note that if $z \in \mathbb{R}^{n+1}$ is such that $z \cdot e_{n+1}>0$, then

$$
z \in C \quad \text { if and only if }\left.\quad z\right|_{\mathbb{R}^{n}} / z \cdot e_{n+1} \in-\sqrt{n} Z_{\infty},
$$

where $\left.z\right|_{\mathbb{R}^{n}}$ denotes the orthogonal projection of $z$ onto $\mathbb{R}^{n}$. We now show that $T\left(\mathbb{R}^{n+1}\right) \subset C$. To see this, note that from (20) and definition (7) it follows that

$$
\left.T y\right|_{\mathbb{R}^{n}}=-\frac{\sqrt{n+1}}{\sqrt{n}} \int_{S^{n-1}} u \phi(y \cdot s(u)) d Z(u),
$$

while the $(n+1)$-st component of $T y$ is given by

$$
T y \cdot e_{n+1}=\frac{\sqrt{n+1}}{n} \int_{S^{n-1}} \phi(y \cdot s(u)) d Z(u),
$$

or equivalently by

$$
T y \cdot e_{n+1}=\frac{1}{\sqrt{n+1}} \int_{S^{n}} \phi(y \cdot w) d \bar{Z}(w) .
$$


Since $\phi>0$, it follows from (23) and (24), together with (6), that

$$
-\left.\frac{1}{\sqrt{n}} T y\right|_{\mathbb{R}^{n}} / T y \cdot e_{n+1} \in \operatorname{int} Z_{\infty}
$$

and hence from (22) we have

$$
T\left(\mathbb{R}^{n+1}\right) \subset C .
$$

From (19) it follows that $d T$, the differential of $T$, is given by

$$
d T(y)=\int_{S^{n}} w \otimes w \phi^{\prime}(y \cdot w) d \bar{Z}(w)
$$

for each $y \in \mathbb{R}^{n+1}$. Since $\phi^{\prime}>0$ and $\bar{Z}$ is not concentrated on a proper subspace of $\mathbb{R}^{n+1}$, we conclude from (27) that the matrix $d T(y)$ is positive definite for each $y \in \mathbb{R}^{n+1}$. Hence, an application of the mean value theorem shows that the transformation $T: \mathbb{R}^{n+1} \rightarrow C$ is globally 1-1.

From (9), (18), (10), (27) together with the Ball-Barthe inequality (25), making the change of variables $z=T y$ and using (26), and (21), we have

$$
\begin{aligned}
\pi^{\frac{n+1}{2}} & =\int_{\mathbb{R}^{n+1}} e^{-|y|^{2}} d y \\
& =\int_{\mathbb{R}^{n+1}} \exp \left\{-\int_{S^{n}}|y \cdot w|^{2} d \bar{Z}(w)\right\} d y \\
& =\int_{\mathbb{R}^{n+1}} \exp \left\{-\int_{S^{n}}\left(\phi(y \cdot w)-\log \phi^{\prime}(y \cdot w)-\log \sqrt{\pi}\right) d \bar{Z}(w)\right\} d y \\
& =\pi^{\frac{n+1}{2}} \int_{\mathbb{R}^{n+1}} \exp \left\{-\int_{S^{n}} \phi(y \cdot w) d \bar{Z}(w)\right\} \exp \left\{\int_{S^{n}} \log \phi^{\prime}(y \cdot w) d \bar{Z}(w)\right\} d y \\
& \leq \pi^{\frac{n+1}{2}} \int_{\mathbb{R}^{n+1}} \exp \left\{-\int_{S^{n}} \phi(y \cdot w) d \bar{Z}(w)\right\}|d T(y)| d y \\
& =\pi^{\frac{n+1}{2}} \int_{\mathbb{R}^{n+1}} \exp \left\{-\sqrt{n+1} T y \cdot e_{n+1}\right\}|d T(y)| d y \\
& \leq \pi^{\frac{n+1}{2}} \int_{C} \exp \left\{-\sqrt{n+1} z \cdot e_{n+1}\right\} d z \\
& \leq \pi^{\frac{n+1}{2}} \int_{0}^{\infty} \int_{\left(-r \sqrt{n} Z_{\infty}\right)} e^{-\sqrt{n+1} r} d x d r \\
& =\pi^{\frac{n+1}{2}} n^{\frac{n}{2}}\left|Z_{\infty}\right| \int_{0}^{\infty} r^{n} e^{-\sqrt{n+1} r} d r \\
& =\pi^{\frac{n+1}{2}} n^{\frac{n}{2}} n !(n+1)^{-(n+1) / 2}\left|Z_{\infty}\right| .
\end{aligned}
$$

Suppose there is equality in the inequality of our theorem. Since $\bar{Z}$ is not concentrated on a proper subspace of $\mathbb{R}^{n+1}$, there are linearly independent unit vectors $w_{1}, \ldots, w_{n+1} \in \operatorname{supp} \bar{Z}$. Assume $w_{0}$ is a different unit vector in $\operatorname{supp} \bar{Z}$. Write $w_{0}=\lambda_{1} w_{1}+\cdots+\lambda_{n+1} w_{n+1}$. At least one coefficient, say $\lambda_{1}$, is not zero. Since $w_{0}, w_{2}, \ldots, w_{n+1}$ are linearly independent, the equality conditions of the Ball-Barthe inequality imply that

$$
\phi^{\prime}\left(y \cdot w_{1}\right) \cdots \phi^{\prime}\left(y \cdot w_{n+1}\right)=\phi^{\prime}\left(y \cdot w_{0}\right) \phi^{\prime}\left(y \cdot w_{2}\right) \cdots \phi^{\prime}\left(y \cdot w_{n+1}\right)
$$

for all $y \in \mathbb{R}^{n+1}$. Since $\phi^{\prime}>0$, we have

$$
\phi^{\prime}\left(y \cdot w_{1}\right)=\phi^{\prime}\left(y \cdot w_{0}\right)
$$


for all $y \in \mathbb{R}^{n+1}$.

Since $\phi^{\prime}$ is not constant, there are $c_{1}$ and $c_{0}$ so that $\phi^{\prime}\left(c_{1}\right) \neq \phi^{\prime}\left(c_{0}\right)$. Since $\bar{Z}$ is supported in an open hemisphere of $S^{n}$, the unit vectors $w_{0}$ and $w_{1}$ are not parallel, and therefore there exists $y \in \mathbb{R}^{n+1}$ such that

$$
y \cdot w_{1}=c_{1}, \quad \text { and } \quad y \cdot w_{0}=c_{0},
$$

producing a contradiction.

Thus equality in the inequality implies that $\operatorname{supp} \bar{Z}=\left\{w_{1}, \ldots, w_{n+1}\right\}$. Since $\bar{Z}$ is isotropic, $w_{1}, \ldots, w_{n+1}$ are orthogonal, and thus, $\operatorname{supp} Z$ consists of the vertices of a regular simplex inscribed in $S^{n-1}$.

After a copy of this paper was communicated to him, Barthe [6] showed how these inequalities could also be obtained from a new "continuous" version of the Brascamp-Lieb inequality.

\section{REFERENCES}

[1] K. Ball, Shadows of convex bodies, Trans. Amer. Math. Soc. 327 (1991), 891-901.

[2] K. Ball, Volume ratios and a reverse isoperimetric inequality, J. London Math. Soc. 44 (1991), 351-359.

[3] K. Ball, Volumes of sections of cubes and related problems, Geometric Aspects of Functional Analysis (J. Lindenstrauss and V.D. Milman, eds.), Springer Lecture Notes in Math., vol. 1376, 1989, pp. 251-260.

[4] F. Barthe, Inégalités fonctionnelles et géométriques obtenues par transport des mesures, Ph.D. thesis, Université de Marne-la-Vallée, 1997.

[5] F. Barthe, On a reverse form of the Brascamp-Lieb inequality, Invent. Math. 134 (1998), 335-361.

[6] F. Barthe, A continuous version of the Brascamp-Lieb inequalities, Geometric aspects of functional analysis, Lecture Notes in Math., vol. 1850, Springer, Berlin, 2004, pp. 53-63.

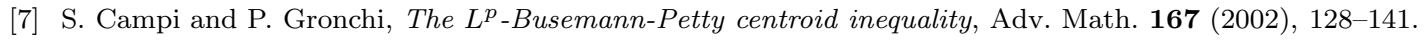

[8] S. Campi and P. Gronchi, On the reverse $L^{p}$-Busemann-Petty centroid inequality, Mathematika 49 (2002), 1-11.

[9] K.-S. Chou and X.-J. Wang, The $L_{p}$-Minkowski problem and the Minkowski problem in centroaffine geometry, Adv. Math., (in press).

[10] R. J. Gardner, The Brunn-Minkowski inequality, Bull. Amer. Math. Soc. 39 (2002), 355-405.

[11] R. J. Gardner, Geometric Tomography, Cambridge Univ. Press, New York, 1995.

[12] A. A. Giannopoulos and V. D. Milman, Extremal problems and isotropic positions of convex bodies, Israel J. Math. 117 (2000), 29-60.

[13] P. Guan and C.-S. Lin, On equation $\operatorname{det}\left(u_{i j}+\delta_{i j} u\right)=u^{p} f$ on $S^{n}$, (preprint).

[14] C. Hu, X.-N. Ma, and C. Shen, On the Christoffel-Minkowski problem for Firey's p-sum, Calc. Var. Partial Differential Equations 21 (2004), 137-155.

[15] D. Hug, E. Lutwak, D. Yang, G. Zhang, On the $L_{p}$ Minkowski problem for polytopes, Discrete Comput. Geom. 33 (2005), 699-715.

[16] M. Ludwig, Valuations of polytopes containing the origin in their interiors, Adv. Math. 170 (2002), 239-256.

[17] M. Ludwig, Ellipsoids and matrix valued valuations, Duke Math. J. 119 (2003), 159-188.

[18] M. Ludwig, Minkowski valuations, Trans. Amer. Math. Soc. 357 (2005), 4191-4213.

[19] E. Lutwak, The Brunn-Minkowski-Firey theory. I. Mixed volumes and the Minkowski problem, J. Differential Geom. 38 (1993), 131-150.

[20] E. Lutwak, The Brunn-Minkowski-Firey theory. II. Affine and geominimal surface areas, Adv. Math. 118 (1996), $244-294$.

[21] E. Lutwak and V. Oliker, On the regularity of solutions to a generalization of the Minkowski problem, J. Differential Geom. 41 (1995), 227-246.

[22] E. Lutwak, D. Yang, and G. Zhang, A new ellipsoid associated with convex bodies, Duke Math. J. 104 (2000), 375-390.

[23] E. Lutwak, D. Yang, and G. Zhang, $L_{p}$ affine isoperimetric inequalities, J. Differential Geom. 56 (2000), 111-132.

[24] E. Lutwak, D. Yang, and G. Zhang, The Cramer-Rao inequality for star bodies, Duke Math. J. 112 (2002), 59-81.

[25] E. Lutwak, D. Yang, and G. Zhang, Sharp affine $L_{p}$ Sobolev inequalities, J. Differential Geom. 62 (2002), 17-38.

[26] E. Lutwak, D. Yang, and G. Zhang, On the $L_{p}$ Minkowski problem, Trans. Amer. Math. Soc. 356 (2004), 4359-4370.

[27] E. Lutwak, D. Yang, and G. Zhang, $L_{p}$ John ellipsoids, Proc. London Math. Soc. 90 (2005), 497-520.

[28] E. Lutwak, D. Yang, and G. Zhang, Volume inequalities for subspaces of $L_{p}$, J. Differential Geom. 68 (2004), $159-184$.

[29] M. Meyer and E. Werner, On the p-affine surface area, Adv. Math. 152 (2000), 288-313.

[30] D. Ryabogin and A. Zvavitch, On the Firey projections of convex bodies, Indiana U. Math. J. 53 (2004), 667-682.

[31] C. Schütt and E. Werner, Polytopes with vertices chosen randomly from the boundary of a convex body, GAFA Seminar Notes 2002, Lecture Notes in Mathematics, Springer-Verlag, 2002, pp. 241-422.

[32] C. Schütt and E. Werner, Surface bodies and p-affine surface area, Adv. Math. 187 (2004), 98-145.

[33] R. Schneider, Convex Bodies: the Brunn-Minkowski Theory, Cambridge Univ. Press, Cambridge, 1993.

[34] A. Stancu, The discrete planar $L_{0}$-Minkowski problem, Adv. Math. 167 (2002), 160-174.

[35] A. Stancu, On the number of solutions to the discrete two-dimensional $L_{0}$-Minkowski problem, Adv. Math. 180 (2003), 290-323. 
[36] A.C. Thompson, Minkowski Geometry, Cambridge Univ. Press, Cambridge, 1996.

[37] V. Umanskiy, On solvability of the two dimensional $L_{p}$-Minkowski problem, Adv. Math. 180 (2003), $176-186$. 